Modelling, Analysis and Simulation

MAS Computational fluid dynamics: science and tool

B. Koren

Report MAS-E0602 January 2006 
Centrum voor Wiskunde en Informatica (CWI) is the national research institute for Mathematics and Computer Science. It is sponsored by the Netherlands Organisation for Scientific Research (NWO).

CWI is a founding member of ERCIM, the European Research Consortium for Informatics and Mathematics.

CWI's research has a theme-oriented structure and is grouped into four clusters. Listed below are the names of the clusters and in parentheses their acronyms.

Probability, Networks and Algorithms (PNA)

Software Engineering (SEN)

\section{Modelling, Analysis and Simulation (MAS)}

Information Systems (INS)

Copyright (C) 2006, Stichting Centrum voor Wiskunde en Informatica

P.O. Box 94079, 1090 GB Amsterdam (NL)

Kruislaan 413, 1098 SJ Amsterdam (NL)

Telephone +31205929333

Telefax +31205924199

ISSN 1386-3703 


\title{
Computational fluid dynamics: science and tool
}

\author{
ABSTRACT \\ The year 2003 marked the 100th anniversary of both the birth of John von Neumann and the \\ first manned flight with a power plane. In the current paper, from a Dutch perspective, attention \\ is paid to the great importance of both events for computational fluid dynamics in general and \\ computational aerodynamics in particular.
}

2000 Mathematics Subject Classification: 01-08; 65-03

Keywords and Phrases: history; computational fluid dynamics 



\title{
Computational Fluid Dynamics: Science and Tool
}

\author{
Barry Koren*
}

The year 2003 marked the 100th anniversary of both the birth of John von Neumann and the first manned flight with a power plane. In the current paper, from a Dutch perspective, attention is paid to the great importance of both events for computational fluid dynamics in general and computational aerodynamics in particular.

\section{Introduction}

The science in which flows of gases and liquids are studied is fluid dynamics, a subdiscipline of physics. No courses in fluid dynamics are given in high school as it requires too much mathematical background. Fluid dynamics is taught at university, at engineering colleges to an advanced level, since one cannot ignore fluid dynamics if one wants to design an aircraft, a rocket, a combustion engine or an artificial heart.

Particularly for aircraft design, knowledge and understanding of fluid dynamics - aerodynamics in this case - is of major importance. Except for the dangerous gravity, all forces acting on a flying plane are forces exerted by air. Safely flying an aircraft over our heads (tanked up with fuel and with passengers on board), makes a precise knowledge, understanding and also control of these aerodynamic forces of crucial importance, in fact a matter of life and death. Moreover, flying must not only be safe, but also fuel-efficient and quiet. For aerospace engineering, aerodynamics is indispensable.

Nowadays, both experimental and theoretical means are available for investigating fluid flows. Wind tunnels are the canonical tool for experimental aerodynamics. The Wright brothers, who made the first manned flight with a power plane (Fig. 1), already had at their disposal a wind tunnel, a self-made one. Wind-tunnel testing has many drawbacks but is deemed trustworthy because real air is used and not virtual air as used in theoretical aerodynamics.

\section{A brief history of computational fluid dynamics}

Nowadays, the technological relevance of theoretical aerodynamics, of theoretical fluid dynamics in general, is widely appreciated. However, in the past it was mainly an academic activity, with results which strongly differed from experimental observations. The technical applications of fluid dynamics first developed independently of theory. Yet, theoretical and technological breakthroughs have closed the gap between theory and practice, and today we see a fruitful interaction between the two. The airplane has played a very stimulating role in this development.

I proceed by highlighting some key developments from the history of theoretical fluid dynamics with an eye towards computational fluid dynamics.

\subsection{Revolutionary innovations}

Theoretical fluid dynamics has an illustrious history $[1,2,3]$. In the course of centuries, many great names have contributed to the understanding of fluid flow and have helped in building up theoretical fluid dynamics, step by step. Theoretical fluid dynamics goes way back to Aristotle (384-322 BC), who introduced the concept of a continuous medium. In my opinion though, it actually began 2000 years later, when Leonhard Euler published his equations of motion for the flow of liquids and gases, on the basis of Newton's second law of motion $[4,5]$.

Euler's idea to describe the motion of liquids and gases in the form of partial differential equations was a revolutionary innovation. However, these equations, known today as the Euler equations, were still unsuited for practical applications, because they neglect friction forces. (Only pressure forces were taken into account.) It was almost a century later, in 1845, that George Stokes proposed fluid-flow equations

${ }^{*}$ CWI, P.O. Box 94079, 1090 GB Amsterdam, the Netherlands (barry.koren@cwi.nl), and Delft University of Technology, Faculty of Aerospace Engineering, P.O. Box 5058, 2600 GB Delft, the Netherlands. 


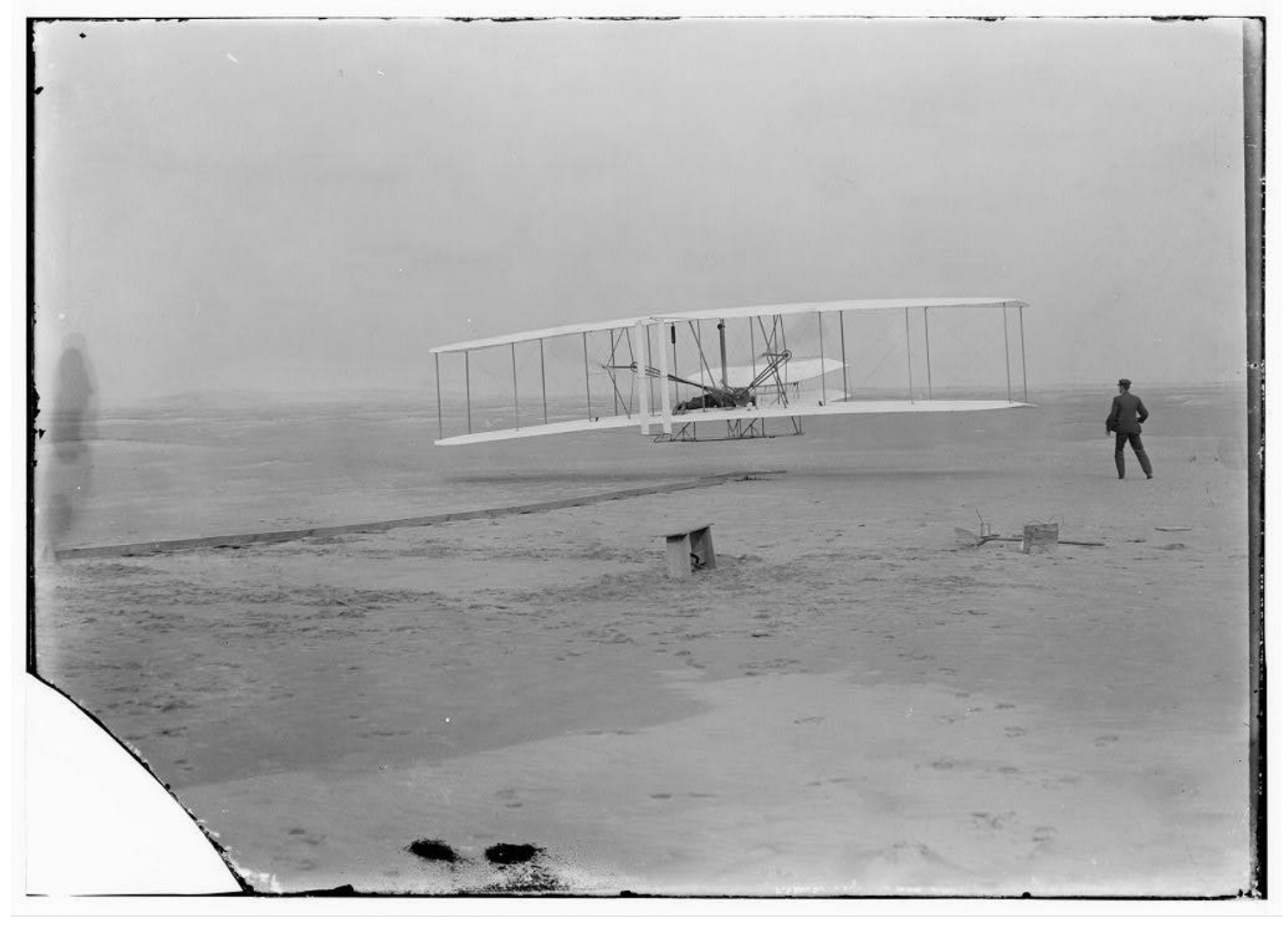

Figure 1: Glass-plate photo of the first manned flight with a power plane (flight distance: 37 meters, flight time: 12.5 seconds), Kitty Hawk, North Carolina, 10.35 h., December 17, 1903. Prone on the lower wing: Orville Wright. Running along with the plane to balance it by hand if necessary: Wilbur Wright. Visible in the foreground: the rail from which the plane took off and the bench on which the wing rested. The very same day, the Wright brothers made a flight of 260 meters and 59 seconds!

which also consider friction [6]; equations, which for an incompressible flow, had already been found by Claude Navier [7] and are now known as the Navier-Stokes equations. With the introduction of the Navier-Stokes equations, the problem of understanding and controlling a large class of fluid flows seemed to be within reach, as it had been reduced to the integration of a handful of fundamental differential equations.

Although formulating the Navier-Stokes equations constituted great progress, the analytical solution of the complete equations appeared to be too ambitious. (The complete analytical understanding still remains one of the outstanding open mathematical problems of the 21st century.) The consequence was the development of a large number of simplified equations, derived from the Navier-Stokes equations for special cases, equations which could be handled analytically. Moreover, a gap continued to exist between experimental and theoretical fluid dynamics. The former developed greatly during the industrial revolution, independently of the latter. It was paradoxical to see that the introduction of the NavierStokes equations led to a further fragmentation into different flow models, models which all described the flow of the same fluid (air in our case); a theoretically highly undesirable situation. Theoretical fluid dynamics stagnated along a front of nonlinear problems. This barrier was finally broken in the second half of the 20th century, with numerical mathematics, at the expense of much, often very much, computational work. A key role was played at this by a Hungarian-born mathematician.

\section{$2.2 \quad$ Trail-blazing ideas from Princeton}

In the tens and twenties of the past century, Budapest was a fruitful breeding ground for scientific talent. There, in 1903, the cradle of John von Neumann (Fig. 2) stood.

In his childhood John von Neumann got private education. At the age of 10 he went to school for the first time, directly to high school. There his great talent for mathematics was discovered. He received extra lessons from mathematicians of the University of Budapest, among them was Fekete, with whom 


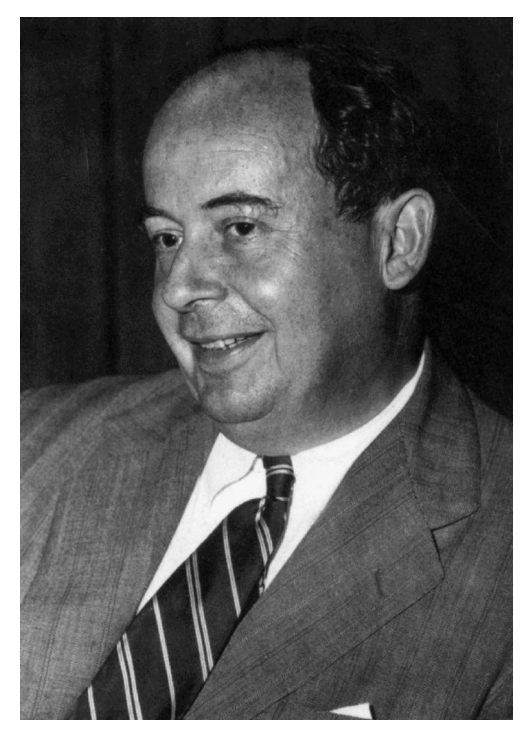

Figure 2: John von Neumann, 1903-1957.

Von Neumann wrote his first mathematics paper, at the age of 18. At that time, in fact, he was already a professional mathematician. Von Neumann studied at the ETH Zürich and the University of Budapest and obtained his $\mathrm{PhD}$ degree at the age of 22 . Next he moved to Germany where he lectured at universities in Berlin and Hamburg. There he was particularly active in pure mathematics: in set theory, algebra, measure theory, topology and group theory. He contributed to existing work, the way to quick recognition. From the mid thirties, Von Neumann chose for a riskier way of working: breaking new ground. He chose for applied mathematics in the sense of mathematicians like Hilbert and Courant, i.e., not mathematics applied to all kinds of ad hoc problems, but the systematic application of mathematics to other sciences, in particular to physics with its subdisciplines like aerodynamics. The fast deteriorating political situation in Europe, from where Von Neumann had already emigrated to Princeton in the beginning of the thirties ${ }^{1}$, played a role in this choice. War was looming; an increasing demand arose for answers to questions from military engineering.

Whereas Von Neumann worked on a mathematical basis for the equations of quantum mechanics before the war, during the war he even 'lowered' himself to developing numerical solution methods for the Euler equations. Very original was his idea to compute possible discontinuities in solutions of the Euler equations without explicitly imposing jump relations. Instead, Von Neumann proposed to introduce artificial (numerical) diffusion, in such a way that the discontinuities automatically appear in a physically correct way: shock capturing, nowadays a standard technique. Meanwhile, he also came up with an original method for analyzing the stability of numerical calculations: a Fourier method, now a standard technique as well.

In 1944, the urgent need arose to apply Von Neumann's numerical methods to automatic calculators, computers, machines that did not yet exist at that time. This urgency motivated Von Neumann to also start working on the development of the computer. In 1944 and 1945 he did trail-blazing work by writing his numerical methods for computing a fluid-flow problem in a set of instructions for a still nonexisting computer. These instructions should not be put into the computer by changing its hardware, say its wiring. Instead, Von Neumann proposed to equip computers with hardware as general as possible, and to store the computing instructions in the computer, together with the other data relevant for the computation (input data, intermediate results and output data). In 1949, the first computer was realized which completely fulfilled Von Neumann's internal programming and memory principles: the EDSAC (Electronic Delay Storage Automatic Calculator), by Wilkes, at Cambridge University. Today, the two principles are still generally applied.

Richardson and Courant \& colleagues already combined theoretical fluid dynamics and numerical mathematics before Von Neumann [9, 10], but still without clear ideas about computers, without computer science. Computational fluid dynamics - shortly CFD - is a combination of three disciplines: theoretical fluid dynamics, numerical mathematics and computer science. Von Neumann brought in this

\footnotetext{
${ }^{1}$ Von Neumann was one of the many scientists who left Europe in the early thirties. Starting 1933, Göttingen ceased to exist as the world center of mathematics, after the Nazi government had imposed strong professional restrictions to scientists of Jewish offspring. For a description of this fall of Göttingen, see Richard Courant's biography [8].
} 
last discipline and may therefore be named the founding father of CFD. A detailed description of Von Neumann's contributions to scientific computing is given by Aspray [11]. A good overview of his other pioneering work can be found in the scientific biography written by Ulam [12].

Traveling from place to place as an honored mathematician, with many social and political obligations, Von Neumann must have had hardly any time to neatly write down his scientific ideas. He only published one paper about both shock capturing and the aforementioned stability analysis and not until 1950 [13]. On his many travels, Von Neumann also visited the Netherlands. In 1954, he was invited speaker during the International Congress of Mathematicians held in Amsterdam. A tea party with Queen Juliana of the Netherlands was arranged for a select group of participants among whom Von Neumann (Fig. 3).

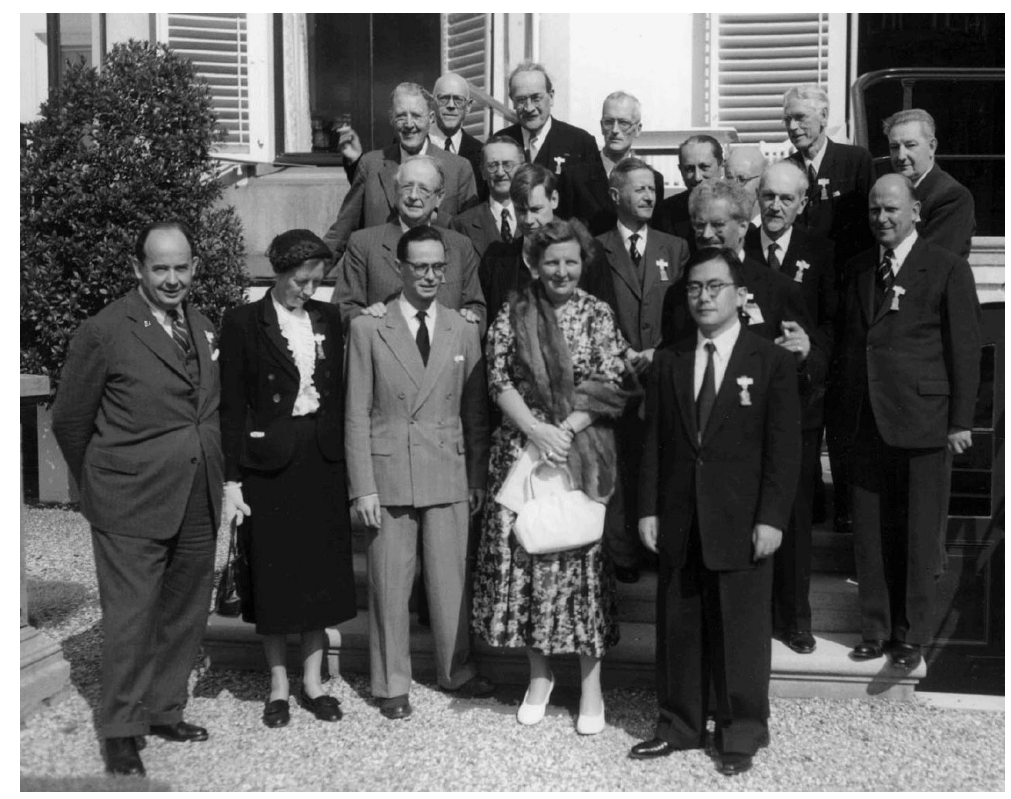

Figure 3: John von Neumann and colleagues at Soestdijk Palace. John von Neumann front row, far left. Queen Juliana, with white handbag, is flanked by the two new recipients of the Fields Medal: Jean-Pierre Serre (with Herman Weyl's hands on his shoulders) and Kunihiko Kodaira. (photo: CWI).

\section{$2.3 \quad$ Pioneering work in Amsterdam}

In the third quarter of the 20th century computer science was arising as a new discipline. Initially, the Netherlands played no significant role in the development of computer science, but the country was quickly moving forward. In 1946, the Mathematisch Centrum ( $M C$ ) was founded in Amsterdam. The mission of this new institute was to do pure and applied mathematics research in order to increase "the level of prosperity and culture in the Netherlands and the contributions of the Netherlands to international culture". (Not at all the pure ivory tower.) The foundation of the MC did not proceed without struggle. The most prominent Dutch mathematician of the day, L.E.J. Brouwer of the University of Amsterdam, was of the opinion that mathematics should be indifferent towards physical sciences and even rejecting of technology; an odd point of view since the work of mathematicians like Hilbert, Courant, Von Neumann and others. With the MC, Brouwer wanted to turn Amsterdam into the new Göttingen of pure mathematics. It did not work out like this. According to his biographer, Brouwer has even been sacrificed to the foundation of the MC ([14], p. 479).

The founders of the MC had heard about Von Neumann's ideas about machines which should be able to perform a series of calculations as independently as possible. They wanted the MC to have a computing department in order to develop a computer and to execute advanced computing work. Aad van Wijngaarden (Fig. 4), former student of J.M. Burgers of the Delft University of Technology, was appointed as the first staff member of the MC, in 1947.

That year he made a study tour to visit Von Neumann in Princeton. Van Wijngaarden and his co-workers designed and constructed the first Dutch computer: the ARRA I (Automatische Relais Rekenmachine Amsterdam I, Fig. 5). New computers were designed and built (one per design only), in 1955 exclusively for the Fokker aircraft industries: the FERTA (Fokker Elektronische Rekenmachine Type ARRA). Much human labor was required to perform computations on these first computers. At the MC, this was done 


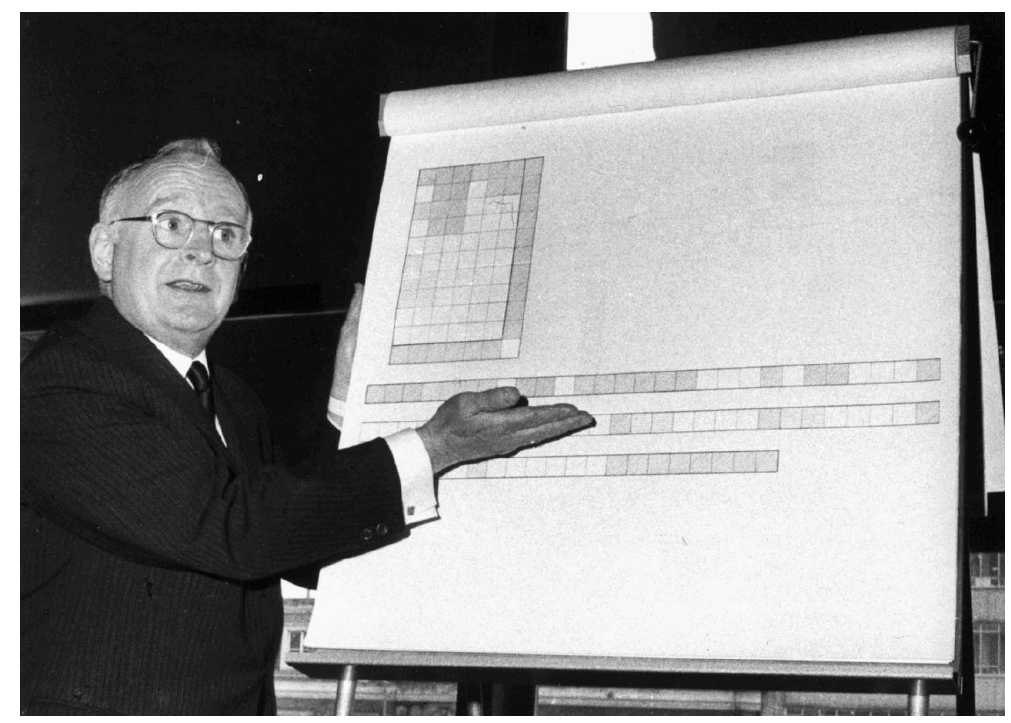

Figure 4: Aad van Wijngaarden, 1916-1987 (photo: CWI).

by young women, schooled in mathematics by Van Wijngaarden. A highlight was the project for the development of the Fokker Friendship airplane, a numerical project on which Van Wijngaarden and his 'computing girls' worked from 1949 until 1951. The computations concerned oscillations of the airplane's wing in subsonic flow: flutter. The first computing work was still very 'external' and bound by machine; for each computation, cables had to be plugged into the computer. With the accomplishment of internal programming as proposed by Von Neumann, the focus of attention shifted entirely to the invention of algorithms and their coding as computer programs. Edsger Dijkstra, a later Turing Award recipient, was appointed at the MC as the first Dutch computer programmer. Van Wijngaarden and Dijkstra left their international mark on computer science with their contributions to the development of the programming language Algol 60 [15] and Van Wijngaarden later again with Algol 68 [16]. In 1979, Van Wijngaarden was awarded an honorary doctorate for his pioneering work by the Delft University of Technology. And the former MC grew into the present $C W I$ (Centrum voor Wiskunde en Informatica), which celebrates its 60th anniversary in 2006.

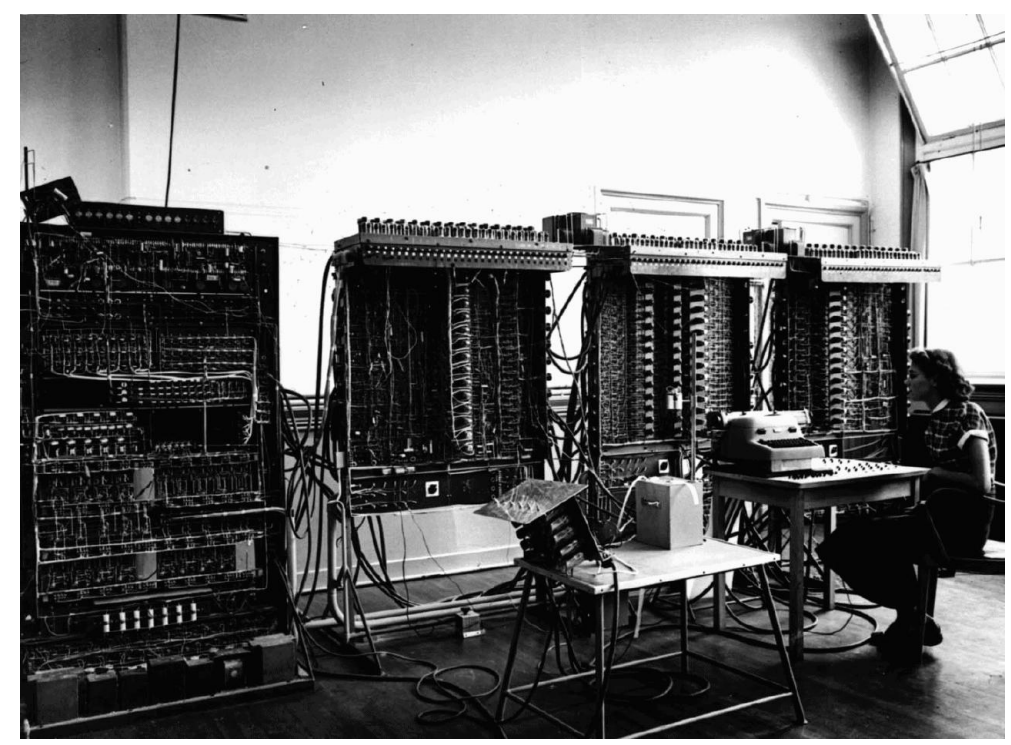

Figure 5: The first computer at the MC and in the Netherlands, the ARRA I in its final set-up. From left to right: power frame and the three arithmetic registers. On the table in the middle: the punch-tape reader. Some 1200 relays are at the back of the machine (photo: CWI). 
CFD research on the basis of the Euler or Navier-Stokes equations, and of the same fundamental character as that established by Von Neumann, was not done in the Netherlands of 1945-1960. For this fundamental work, we have to go to the United States and the Sowjet-Union of the fifties.

\subsection{A continuous flow of CFD from New York}

In the beginning of December 1941, a passenger ship carried a 15-year old Hungarian boy from Europe to the United States. The boy, along with his parents, was escaping the tragic fate waiting for many European Jews, and was carrying with him two letters of recommendation from his teachers. (It was to be the last passenger ship from Europe's mainland to the United States in the years to come. During the trip, the United States was drawn into the Second World War by the attack on Pearl Harbor.) It seems likely that Von Neumann saw the letters brought by his young fellow-countryman, for the boy, Peter Lax (Fig. 6), had a meteoric rise to success. In 1945, while still a teenager, he became involved in the Manhattan project, in 1949, he received his PhD degree from New York University, with Richard Courant as his thesis advisor, and in 1951, he became assistant professor there. His work in mathematics continues to this day and has led to many honors and awards, among these the 2005 Abel Prize in mathematics $[17]$.

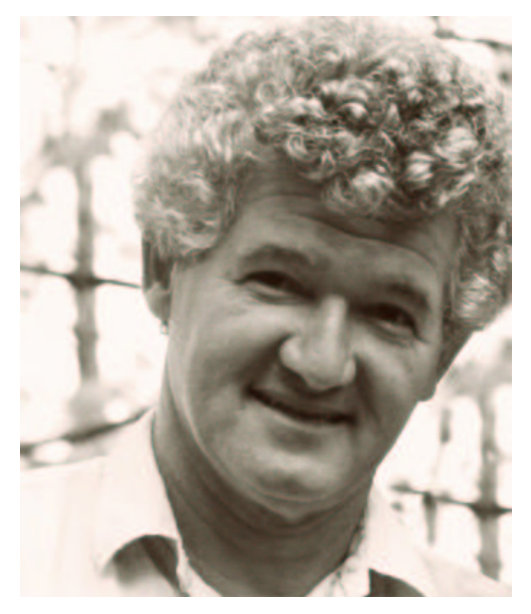

Figure 6: Peter D. Lax.

Like Von Neumann, Lax is a homo universalis in mathematics. He has performed ground breaking research, but was also a very productive and versatile author of mathematics books. He wrote books on such diverse topics as partial differential equations, scattering theory, linear algebra and functional analysis. He is most well known though for his research on numerical methods for partial differential equations, in particular for hyperbolic systems of conservation laws, such as those arising in fluid dynamics. Lax's name has been given to several mathematical discoveries of importance to CFD. Examples are:

- the Lax equivalence theorem [18], stating that consistency and stability of a finite-difference discretization of a well-posed initial-boundary-value problem are necessary and sufficient for the convergence of that discretization,

- the Lax-Friedrichs scheme [19], a stabilized central finite-difference scheme for hyperbolic partial differential equations,

- the Lax-Wendroff scheme [20], a more accurate, but equally stable version of the Lax-Friedrichs scheme,

- the Lax entropy condition [21], a principle for selecting the unique, physically correct shock-wave solution of nonlinear, hyperbolic partial differential equations, that allow multiple shock-wave solutions, and

- the Harten - Lax - Van Leer scheme [22], a very efficient numerical method for solving the Riemann problem. 
Like Von Neumann, Lax was (and still is) a strong proponent of the use of computers in mathematics. A quote of Lax is: "The impact of computers on mathematics (both applied and pure) is comparable to the roles of telescopes in astronomy and microscopes in biology".

Despite the Second World War and the Cold War, Lax has always had very good connections worldwide with scientists. One such relation is with the Russian mathematician mentioned in the next section.

\subsection{A brilliant idea from Moscow}

A substantial part of the Euler and Navier-Stokes software used worldwide, is based on a single journal paper [23], distilled by the then young Russian mathematician Sergei Konstantinovich Godunov (Fig. 7), from his $\mathrm{PhD}$ thesis.

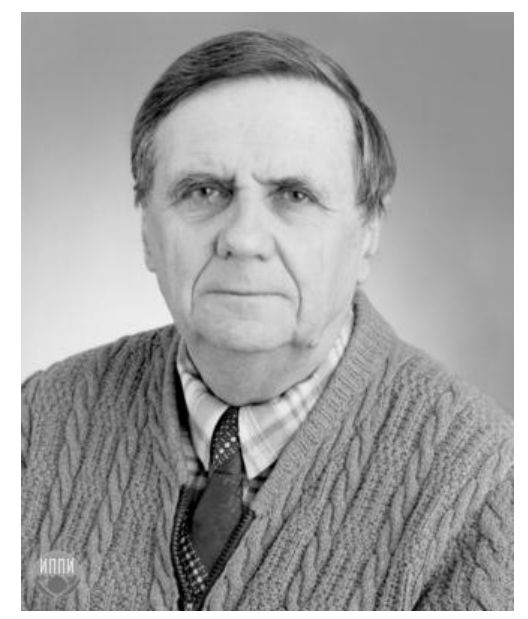

Figure 7: Sergei Konstantinovich Godunov.

Godunov proposed the following. Suppose one has a tube and in it a membrane separating a gas on the left with uniformly constant pressure, from a gas on the right with a likewise uniformly constant but lower pressure (Fig. 8, above). If the membrane is instantaneously removed - the traffic light changes from red to green - then the yellow gas will push the blue gas to the right; the interface between the two gases, the contact discontinuity, runs to the right. At the same time, two pressure waves start running through the tube: a compression wave running ahead of the contact discontinuity and an expansion wave running to the left (Fig. 8, below). In the 19th century, the Euler flow in this tube, a shock tube, had already been computed by Riemann, with 'pencil and paper' [24]. (For this old work of Riemann, Duivesteijn has written a nice, interactive Java applet [25].) For the computation of the flow in a tube in the case of an initial condition which has more spatial variation, Godunov proposed to decompose the tube into virtual cells (Fig. 9, above), with per cell a uniformly constant gas state, and with each individual cell wall to be considered as the aforementioned membrane (traffic light). To know the interaction between the gas states in two neighboring cells, one instantaneously 'removes' the cell wall separating the two cells, computes the Riemann solution locally there, and hence the local mass, momentum and energy flux (Fig. 9, below). This is done at all cell faces. With this, the net transport for each cell is known and a time step can be made. A plain method and a very simple flow problem, so it seems. If one can do this well, the flow around a complete aircraft or spacecraft can be computed. The remarkable property of the method is that at the lowest discrete level, that of cell faces, a lot of physics has been built into it, not just numerical mathematics.

The more cells, the better the accuracy, yet also the more expensive the computation. Godunov did not have the disposal of computers, but of 'computing girls', who called Godunov and his colleague $\mathrm{PhD}$ students 'that science', and who received payment on the basis of the amount of computations they performed, right or wrong. No real CFD either!

In 1997, Godunov received an honorary doctorate from the University of Michigan and a symposium was organized for him at the university's Department of Aerospace Engineering. At this symposium, in a one-and-a-half hour lecture, Godunov gave insight into his earlier research, work of which one failed to see the strategic importance in the Sowjet-Union of those days. The lecture, a historic event, has been published $[26,27]$. 
A second important result in Godunov's classical paper from 1959 [23] is his proof that it is impossible to devise a linear method which is more than first-order accurate, without being plagued by physically incorrect oscillations in the solution: wiggles (Fig. 10). With a first-order accurate method, the solution becomes twice as accurate and remains free of wiggles when the cells are taken twice as small. With a second-order accurate method, the solution becomes four times more accurate then, but - unfortunately - possibly wiggle-ridden.

Wiggles can be very troublesome in practice. E.g., a simple speed-of-sound calculation in a single cell only, may break down the entire flow computation, because of a possibly negative pressure. The wiggle problem does not occur with Godunov's method only, it is a general problem. A drawback of Godunov's method is that it is computing intensive; at each cell face the intricate Riemann problem is solved exactly.
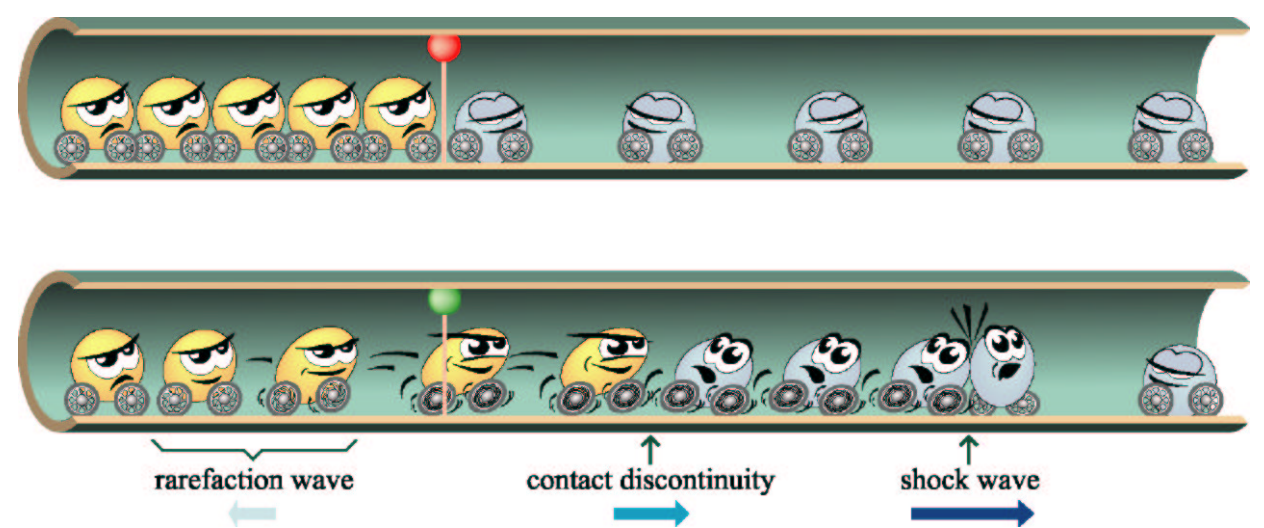

Figure 8: Shock tube. Above: condition of rest in left and right part: high and low pressure, respectively. Below: condition of motion with shock wave and contact discontinuity running to the right and rarefaction wave running to the left. (drawing: Tobias Baanders, CWI).

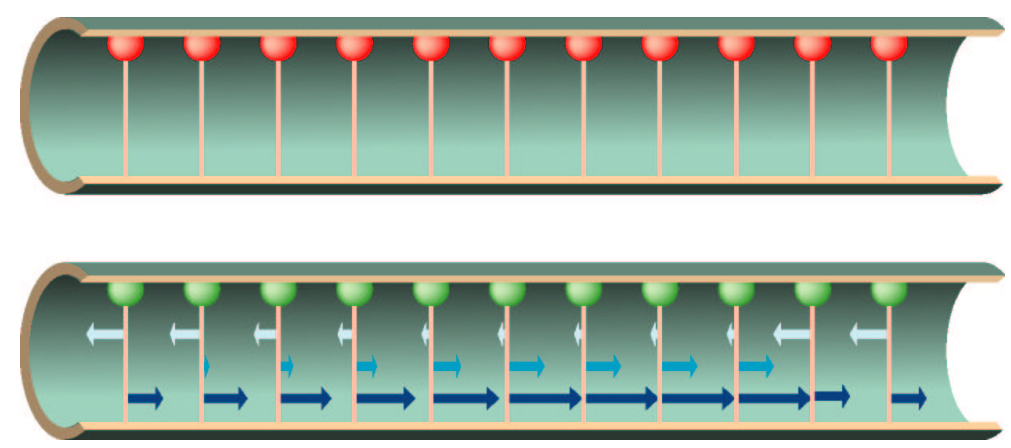

Figure 9: Shock tube divided into small cells. Above: cells. Below: wave propagation over all cell faces. (drawing: Tobias Baanders, CWI).
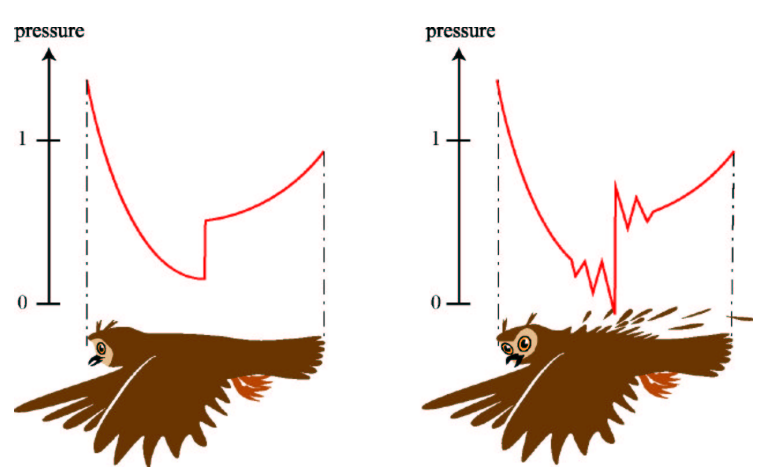

Figure 10: Right and wrong pressure distribution. Left: without wiggles. Right: with wiggles. (drawing: Tobias Baanders, CWI). 


\subsection{Technology pushes from Leiden}

It has taken about two decades before good remedies were found against the wiggles of higher-order methods and the high cost of the Godunov algorithm. The aid came from an astronomer. In the universe, large clouds of hydrogen are found. Simulation of the flow of this hydrogen provides information about the development of galaxies. The literally astronomical speeds and pressures, which may arise in these computations, impose high demands on the accuracy and particularly the robustness of the computational methods to be applied. While still in Leiden, the astronomer Bram van Leer (Fig. 11) published a series of papers in the seventies in which he proposed methods which are second-order accurate and do not allow wiggles. The fifth and last paper in this series is [28]. Furthermore, Van Leer introduced a computationally efficient alternative for the Godunov algorithm [29]. Two technology pushes, not only for astronomy but also for aerospace engineering, as well as for other disciplines. In 1990, Van Leer was awarded an honorary doctorate for this work by the Free University of Brussels.

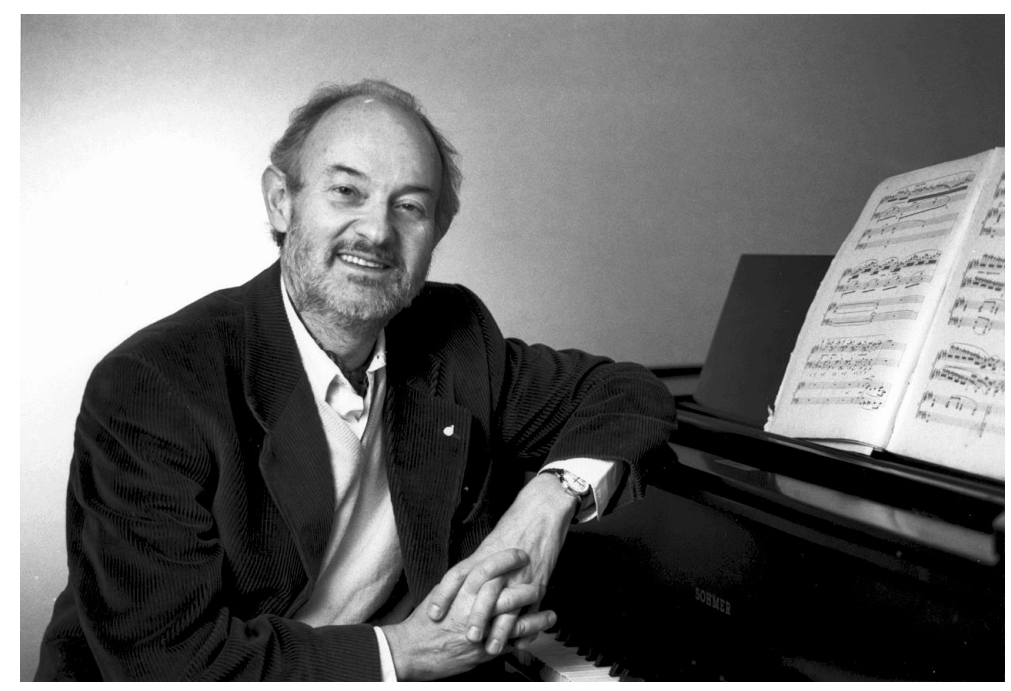

Figure 11: Bram van Leer (photo: Michigan Engineering).

\subsection{Efficient solution algorithms from Rehovot and other places}

How does it work - broadly speaking - an Euler- or Navier-Stokes-flow computation around an aircraft? The airspace around the aircraft may be divided, up to a large distance from the aircraft, into e.g. small hexahedra, small 3D cells. Per cell, just as in the 1D shock tube example, one can then compute the net inflow of mass, momentum and energy, using at each cell face the Godunov-alternative à la Van Leer or other alternatives, like the Roe scheme [30] or the Osher scheme [31]. The finer the knitwear of cells around the aircraft, the grid (Fig. 12), the more accurate the solution, but also the higher the computing cost.

A grid of one million cells for an Euler- or Navier-Stokes-flow computation is not unusual. Suppose that we want to simulate a steady flow. We then have to solve per cell: five coupled, nonlinear partial differential equations. The cells themselves are coupled as well; what flows out of a cell, flows into a neighbor cell (or across a boundary of the computational domain). In Navier-Stokes-flow computations, the flow solution in a single cell may influence the flow solutions in all other cells. In our modest example, we may have to solve a system of five million coupled, nonlinear, algebraic equations. Efficient solution of these millions of equations is an art in itself. Many efficient solution algorithms have been developed, the most efficient of which are the multigrid algorithms. Multigrid methods were invented at different locations and by several people. A leading role has been played by Achi Brandt from the Weizmann Institute of Science in Rehovot, Israel [32]. Multigrid algorithms have a linear increase of the computing time with the number of cells. This may seem expensive: 2, 3 or 4 times higher computing cost for a grid with 2, 3 or 4 times more cells, respectively, but it is not. In numerical mathematics, no bulk discount is given. For many solution algorithms applies: $2^{2}, 3^{2}, 4^{2}, \ldots$ times higher computing cost for a grid with $2,3,4, \ldots$ times more cells! For the interested reader, a book on multigrid methods is [33]. 


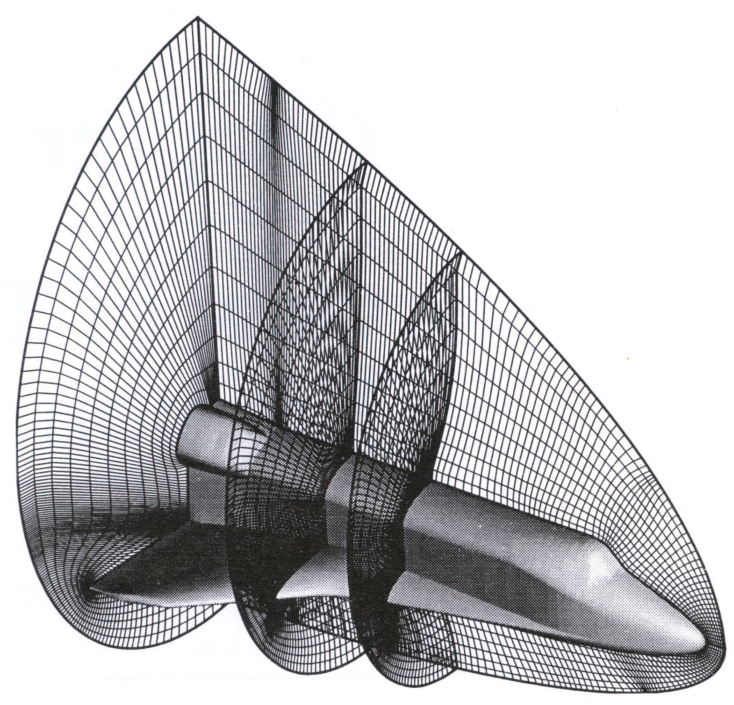

Figure 12: Cross sections of a hexahedral grid around the Space Shuttle.

\section{Present state-of-the-art in CFD}

\subsection{An example}

A quick impression will now be given of what can be done with CFD by means of a standard flow problem. It concerns the recent MSc work of Jeroen Wackers. From scratch, he developed 2D Euler software in which the grid is automatically adapted to the flow and this is one of his results. Consider the channel depicted in Figure 13, shown in it: a uniformly constant supersonic air flow (from left to right) at three times the speed of sound. One may consider the channel to be a stylized engine inlet of a supersonic aircraft. In fact it is just a benchmark geometry [34, 35]. The red vertical valve in the bottom of the channel instantaneously snaps up, such that, together with the red horizontal plate, it forms a step which suddenly chokes part of the channel.

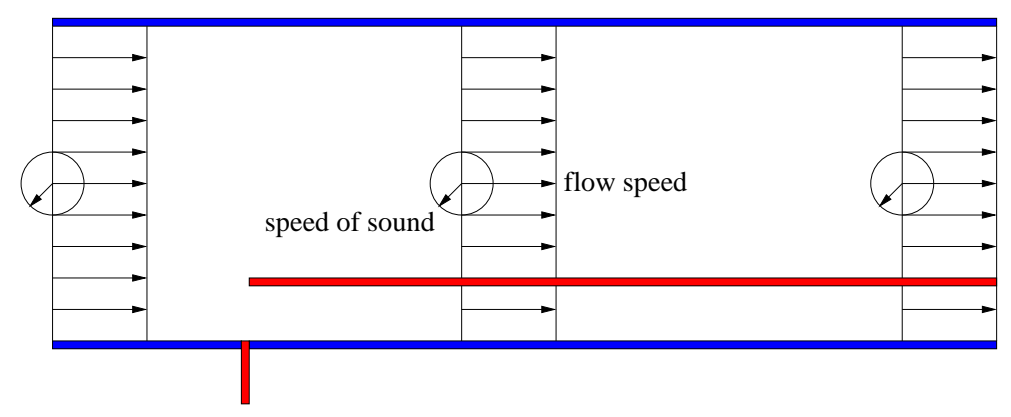

Figure 13: 2D, parallel channel, in it: a parallel plate and a vertical valve which is still open.

Fig. 14 shows a computational result. We see how the uniformly constant initial solution and the grid have developed after some time. The computational method highly satisfies the often conflicting requirements of numerical stability, accuracy and monotonicity on the one hand, and computing and memory efficiency on the other hand [36].

\section{$3.2 \quad$ Books, journals and software}

Twenty years ago, text books on CFD were very rare, but several books are available now (see, e.g., $[37,38,39,40])$. Also, scientific journals on CFD exist. Moreover, ready-for-use CFD software can be bought these days. Each issue of, e.g., the monthly Aerospace America contains some colorful, full-page advertisements for CFD software. A practical overview of CFD literature, software and also vacancies can be found on the website of CFD Online [41]. 

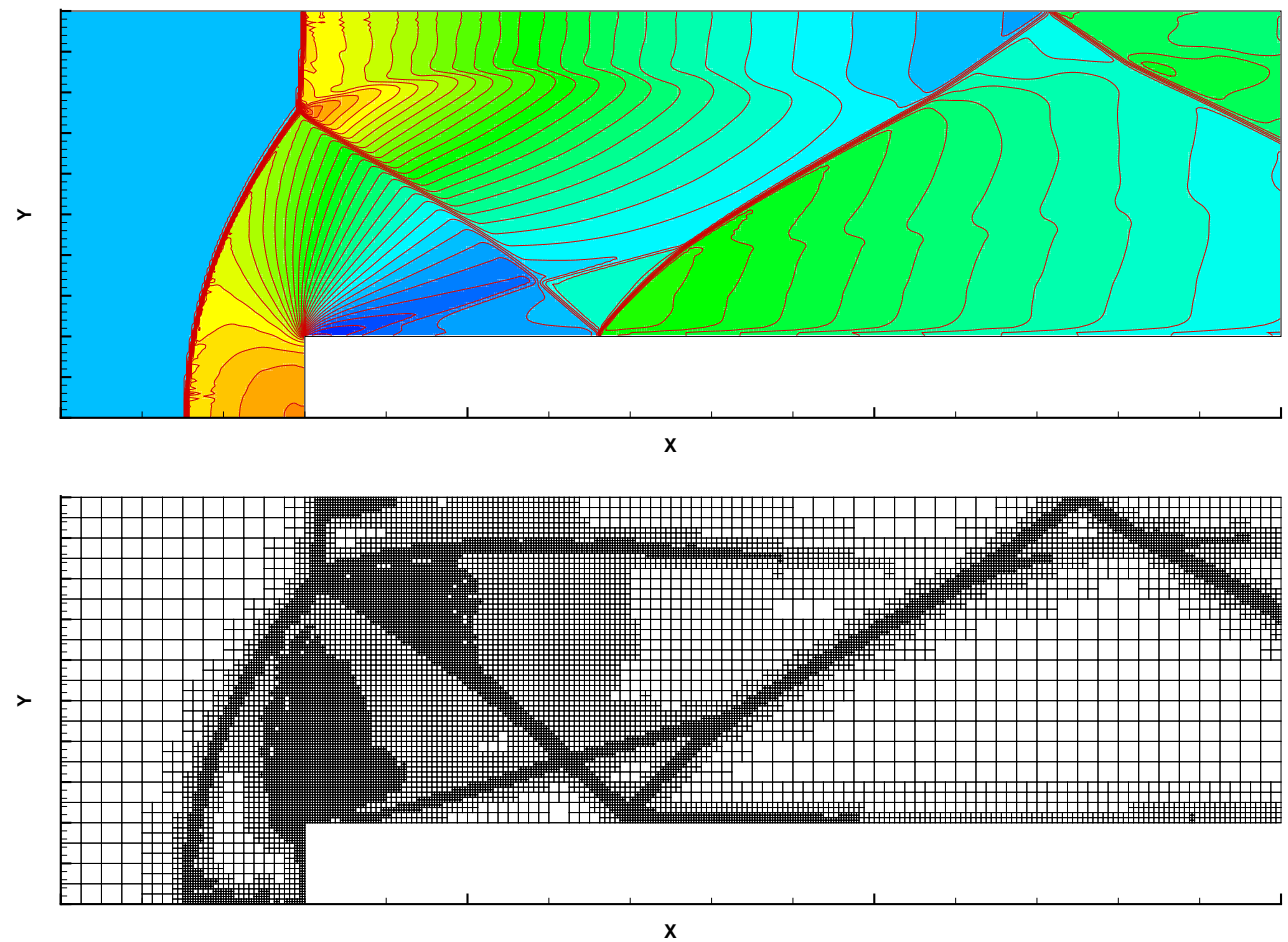

Figure 14: Computational result some time after instantaneously closing lower part of channel. Above: iso-lines of density. When the vertical valve is still in open position, the density in the entire channel is constant (everywhere the same blue color as at the inlet). Below: computational grid automatically adapted to flow solution.

Today, CFD's role is about equally important as that of experimental fluid dynamics. And CFD continues to grow. It is still fed by improvements in both computer science and numerical mathematics. CFD itself stimulates further research in computer science and numerical mathematics; a fruitful interaction.

At present, CFD enters into full cooperation with other disciplines, such as structural mechanics (computational fluid-structure interactions) and electromagnetism (computational magnetohydrodynamics).

\section{Outlook}

The fact that commercial CFD software is a success, is a proof of the practical importance of the theoretical fluid-dynamics work since Euler. The growing availability of CFD software may seem to be a threat for CFD research; CFD researchers seem to make themselves redundant by their own successful work. Yet, this growing software availability may also be considered a good development. It should be avoided that everyone writes his/her own Euler or Navier-Stokes code. Coding such software from scratch gives the best insight and is pleasing work, but may easily take too much time.

\subsection{Education}

How to teach CFD, now that it has become more and more important as easily available, automatic tool? Not just factual knowledge but also understanding of the mathematical and physical principles of CFD is and remains to be indispensable, not only when practicing it as a science, but also when using it as a tool. The CFD-tool user must know and understand these principles well in: (i) posing the computational problem, (ii) choosing the numerical method to solve that problem, and (iii) interpreting the computational results. The user must know the possibilities and limitations of computational methods and should be able to assess whether the obtained computational results fulfill the expectations or not. If not, it should be found out why. CFD is not solving flow problems by blind numerical force, on the contrary. Stimulated by the growing potential of CFD still more complicated flow problems will be considered, problems which will require even more knowledge and understanding of flow physics and numerical mathematics. 


\subsection{Research}

As CFD becomes more and more mature, it also becomes more difficult to contribute to it through fundamental research. Last decades a lot of ground has been cut from under researchers' feet. Think of the fundamental work that Godunov still easily (however?) did as a PhD student. Students will have to acquire an ever growing knowledge and understanding of CFD before they can start working in it themselves. On the other hand, thanks to the availability of CFD tools, the possibilities for application of CFD are far better now than in Godunov's years of study. Just how CFD will develop remains unpredictable, and this is part of what makes it such an exciting and attractive discipline.

Against the somewhat pessimistic prospect that fundamental CFD research is becoming more and more difficult, the attractive perspective stands that in CFD still plenty of research questions exist. New fluid-flow problems will continue to arise, and there will certainly be times when we may say with Orville Wright: "Isn't it astonishing that all these secrets have been preserved for so many years just so that we could discover them!"

\section{References}

[1] T. von Kármán, Aerodynamics, McGraw-Hill, New York, 1963.

[2] H. Rouse And S. Ince, History of Hydraulics, Dover, New York, 1963.

[3] J.D. Anderson, A History of Aerodynamics, Cambridge University Press, Cambridge, 1997.

[4] L. Euler, Principes généraux du mouvement des fluides, Mémoires de l' Académie des Sciences de Berlin, 11 (1755), pp. 274-315.

[5] M.D. SALAs, Leonhard Euler and his contributions to fluid mechanics, AIAA-paper 88-3564, AIAA, Reston, VA, 1988.

[6] G.G. Stokes, On the theories of the internal friction of fluids in motion, and of the equilibrium and motion of elastic solids, Transactions of the Cambridge Philosophical Society, 8 (1845), pp. 287.

[7] C.L.M.H. Navier, Mémoire sur les lois du mouvement des fluides, Mémoires de l'Académie des Sciences, 6 (1822), pp. 389-440.

[8] C. ReID, Courant in Göttingen and New York. The Story of an Improbable Mathematician, Springer, New York, 1976.

[9] L.F. Richardson, Weather Prediction by Numerical Process, Cambridge University Press, Cambridge, 1922.

[10] R. Courant, K.O. Friedrichs And H. Lewy, Über die partiellen Differenzgleichungen der mathematischen Physik, Mathematische Annalen, 100 (1928), pp. 32-74.

[11] W. Aspray, John von Neumann and the Origins of Modern Computing, MIT Press, Cambridge, Massachusetts, 1990.

[12] S. UlAm, John von Neumann, 1903-195\%, Bulletin of the American Mathematical Society, 64 (1958), pp. $1-49$.

[13] J. von Neumann and R.D. Richtmyer, A method for the numerical calculation of shocks, Journal of Applied Physics, 21 (1950), pp. 232-237.

[14] D. van Dalen, L.E.J. Brouwer, 1881-1966, Het Heldere Licht van de Wiskunde, Bert Bakker, Amsterdam, 2002.

[15] P. Naur (ED.), Revised Report on the Algorithmic Language Algol 60 (to be downloaded from http://www . masswerk . at/algol60/report.htm).

[16] A. Van Wijngandden et Al., Revised Report on the Algorithmic Language Algol 68, Springer, Berlin, 1976.

[17] http://www.abelprisen.no/en/. 
[18] P.D. Lax And R.D. Richtmyer, Survey of the stability of linear finite difference equations, Communications on Pure and Applied Mathematics, 9 (1956), pp. 267-293.

[19] P.D. LAx, Weak solutions of nonlinear hyperbolic equations and their numerical computation, Communications on Pure and Applied Mathematics, 7 (1954), pp. 159-193.

[20] P.D. Lax and B. Wendroff, Systems of conservation laws, Communications on Pure and Applied Mathematics, 13 (1960), pp. 217-237.

[21] P.D. Lax, Hyperbolic Systems of Conservation Laws and the Mathematical Theory of Shock Waves, SIAM, Philadelphia, 1973.

[22] A. Harten, P.D. Lax and B. van Leer, On upstream differencing and Godunov-type schemes for hyperbolic conservation laws, SIAM Review, 25 (1983), pp. 35-61.

[23] S.K. Godunov, Finite difference method for the numerical computation of discontinuous solutions of the equations of fluid dynamics, Matematicheskii Sbornik, 47 (1959), pp. 271-306. Translated from Russian at the Cornell Aeronautical Laboratory.

[24] G.F.B. Riemann, Über die Fortpflanzung ebener Luftwellen von endlicher Schwingungsweite, in: Gesammelte Werke, Leipzig, 1876. Reprint: Dover, New York, 1953.

[25] G.F. Duivesteisn, Visual shock tube solver (to be downloaded from http://www.piteon.nl/cfd/).

[26] B. VAN LEER, An introduction to the article "Reminiscences about difference schemes", by S.K. Godunov, Journal of Computational Physics, 153 (1999), pp. 1-5.

[27] S.K. Godunov, Reminiscences about difference schemes, Journal of Computational Physics, 153 (1999), pp. 6-25.

[28] B. VAN LEER, Towards the ultimate conservative difference scheme. V. A second-order sequel to Godunov's method, Journal of Computational Physics, 32 (1979), pp. 101-136. Reprint: Journal of Computational Physics, 135 (1997), pp. 229-248.

[29] B. van LeER, Flux-vector splitting for the Euler equations, in Lecture Notes in Physics, Vol. 170, Springer, Berlin, 1982, pp. 507-512.

[30] P.L. RoE, Approximate Riemann solvers, parameter vectors, and differences schemes, Journal of Computational Physics, 43 (1981), pp. 357-372.

[31] S. Osher And F. Solomon, Upwind difference schemes for hyperbolic systems of conservation laws, Mathematics of Computation, 38 (1982), pp. 339-374.

[32] A. Brandt, Multi-level adaptive solutions to boundary-value problems, Mathematics of Computation, 31 (1977), pp. 333-390.

[33] U. Trottenberg, C.W. Oosterlee and A. Schüller, Multigrid, Academic Press, New York, 2001.

[34] A.F. EMERY, An evaluation of several differencing methods for inviscid fluid flow problems, Journal of Computational Physics, 2 (1968), pp. 306-331.

[35] P.R. Woodward And P. Colella, The numerical simulation of two-dimensional fluid flow with strong shocks, Journal of Computational Physics, 54 (1984), pp. 115-173.

[36] J. Wackers AND B. Koren, A simple and efficient space-time adaptive grid technique for unsteady compressible flows, in Proceedings 16th AIAA CFD Conference (CD-ROM), AIAA-paper 2003-3825, American Institute of Aeronautics and Astronautics, Reston, VA, 2003.

[37] P. Wesseling, Principles of Computational Fluid Dynamics, Springer, Berlin, 2001.

[38] P.J. RoAche, Fundamentals of Computational Fluid Dynamics, Hermosa, Albuquerque, NM, 1998.

[39] Ch. Hirsch, Numerical Computation of Internal and External Flows, Vol. 1 Fundamentals of Numerical Discretization, Vol. 2 Computational Methods for Inviscid and Viscous Flows, Wiley, Chichester, 1988-1990. 
[40] C.A.J. Fletcher, Computational Techniques for Fluid Dynamics, Vol. 1 Fundamental and General Techniques, Vol. 2 Specific Techniques for Different Flow Categories, Springer, Berlin, 1988.

[41] http://www.cfd-online.com.

\section{Contents}

1 Introduction $\quad 1$

2 A brief history of computational fluid dynamics $\quad 1$

2.1 Revolutionary innovations . . . . . . . . . . . . . . . . . . . . . . . . . 1

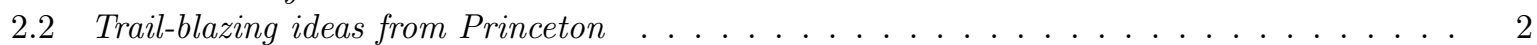

2.3 Pioneering work in Amsterdam . . . . . . . . . . . . . . . . . . . . . . . . . . 4

2.4 A continuous flow of CFD from New York . . . . . . . . . . . . . . . . . . . . . . . . 6

2.5 A brilliant idea from Moscow . . . . . . . . . . . . . . . . . . . . . . 7

2.6 Technology pushes from Leiden . . . . . . . . . . . . . . . . . . . . . . . . . . . . . . . . . 9

2.7 Efficient solution algorithms from Rehovot and other places . . . . . . . . . . . . . . . . . 9

3 Present state-of-the-art in CFD 10

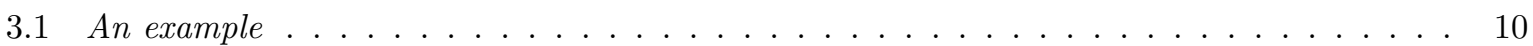

3.2 Books, journals and software . . . . . . . . . . . . . . . . . . . . . 10

4 Outlook $r$

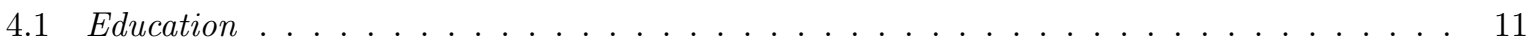

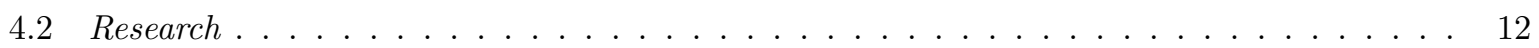

